УДК $517.927+517.984 .52$

Ya. O. Baranetskij, P. I. Kalenyuk, L. I. Kolyasa, M. I. Kopach

\title{
NONLOCAL MULTIPOINT PROBLEM FOR ORDINARY DIFFERENTIAL EQUATION OF EVEN ORDER WITH INVOLUTION
}

\begin{abstract}
Ya. O. Baranetskij, P. I. Kalenyuk, L. I. Kolyasa, M. I. Kopach. Nonlocal multipoint problem for an ordinary differential equations of even order involution, Mat. Stud. 49 (2018), 80-94.

We study a nonlocal multipoint problem for an ordinary differential equation of even order with coefficients containing an involution operator. The spectral properties of a self-adjoint operator with boundary conditions generalizing the conditions of antiperiodicity are investigated. For a differential equation of even order, we consider a problem with multipoint conditions that are perturbations of self-adjoint boundary conditions. We study cases when multipoint conditions include boundary conditions that are regular, but not strongly regular according to Birkhoff, or irregular. The eigenvalues and elements of the system of the root functions of the operator of the problem are determined. It is proved that the system is complete and contains an infinite number of associated functions. Sufficient conditions are obtained for which this system is a Riesz basis. Similar results are obtained for the operator generated by the multipoint problem for an ordinary differential equation of even order with coefficients containing the involution operator.
\end{abstract}

1. Introduction. We will use the following notation. Let

$$
\begin{aligned}
& W_{2}^{2 n}(0,1):=\left\{y \in L_{2}(0,1): y^{(m)} \in C[0,1], y^{(2 n)} \in L_{2}(0,1) ; m=0,1, \ldots, 2 n-1\right\} \\
& \left(y, u ; W_{2}^{2 n}(0,1)\right):=\sum_{k=0}^{2 n}\left(y^{(k)}, u^{(k)} ; L_{2}(0,1)\right),\left\|y ; W_{2}^{2 n}(0,1)\right\|^{2}:=\left(y, y: W_{2}^{2 n}(0,1)\right) ;
\end{aligned}
$$

$W^{*}(0,1)$ is the space of continuous linear functionals over $W_{2}^{2 n}(0,1)$;

$\left[L_{2}(0,1)\right]$ is the algebra of the bounded linear operators $A: L_{2}(0,1) \rightarrow L_{2}(0,1)$;

$I: L_{2}(0,1) \rightarrow L_{2}(0,1)$ is the operator of involution; $I y(x):=y(1-x)$;

$p_{j}:=\frac{1}{2}\left(E+(-1)^{j} I\right)$ are the orthoprojectors of the space $L_{2}(0,1)$;

$H_{j} \equiv\left\{y \in L_{2}(0,1): y=p_{j} y\right\} ; K_{j}:=\left\{e^{i c x}+(-1)^{j} e^{i c(1-x)}, c \in \mathbb{R}\right\}, j=0,1$.

A function from the space $H_{0} \quad\left(H_{1}\right)$ will be called symmetric (antisymmetric), respectively. A boundary condition will be called symmetric if the kernel of the corresponding functional belongs to an arbitrary function from $K_{1}\left(K_{0}\right)$.

2. Statment of the problem. Properties of the system of root functions of boundary value problems are important in constructing solutions of many non-stationary problems by means of the Fourier method or its analogues. For the case of ordinary differential equations, on the

2010 Mathematics Subject Classification: 34K08, 34L10, $46 \mathrm{~B} 15$.

Keywords: differential-operator equation; root function; operator of involution; essentially a non-self-adjoint operator; Riesz basis; nonlocal problem.

doi:10.15330/ms.49.1.80-94

(C) Ya. O. Baranetskij, P. I. Kalenyuk, L. I. Kolyasa, M. I. Kopach , 2018 
finite interval, spectral properties of the system of eigenfunctions of boundary-value problems generated by strongly regular according to Birkhoff boundary conditions is established in [6], [7], [13], [14], [16], [20]-[23].

In the case when the boundary conditions are regular but not strongly regular, in the papers [10], [15], [19] it was established that the system of root subspaces corresponding to the multiple eigenvalues of the boundary value problem forms the Riesz's basis of space of subspaces. In the papers [8], [17] boundary value problems for higher-order differential equations with coefficients containing the involution operator were established. The properties of non-self-adjoint operators determined in an abstract separable Hilbert space were studied in [11].

The paper continues to study the spectral properties of multipoint problems for differential equations of even order (see [1]-[5], [11], [12]) .

Consider the multipoint problem

$$
\begin{gathered}
(-1)^{n} y^{(2 n)}(x)+\sum_{j=1}^{n} a_{j}(I) y(x)=f(x), x \in(0,1) \\
l_{j} y:=y^{(j-1)}(0)-(-1)^{j} y^{(j-1)}(1)+\sum_{s=0}^{r} \sum_{m=0}^{k_{j}} b_{j, m, s} y^{(m)}\left(x_{s}\right)=0, j=1,2, \ldots, n, \\
l_{n+j} y:=y^{(j)}(0)-(-1)^{j} y^{(n+j-1)}(1)=0, j=1,2, \ldots, n,
\end{gathered}
$$

where

$$
\begin{gathered}
a_{j}(I) y:=a_{j}\left(y^{(2 j-1)}(x)-y^{(2 j-1)}(1-x)\right), 0=x_{0}<x_{1}, \ldots, x_{r}<1, \\
a_{j} \in \mathbb{R}, b_{j, m, s} \in \mathbb{R}, m=0,1, \ldots, k_{j}, k_{j}<2 n, s=0,1, \ldots, r, j=1,2, \ldots, n .
\end{gathered}
$$

3. Self-adjoint boundary value problem. We consider the boundary-value problem

$$
\begin{gathered}
(-1)^{n} y^{(2 n)}(x)=f(x), x \in(0,1), \\
l_{0, j} y:=y^{(j-1)}(0)-(-1)^{j} y^{(j-1)}(1)=0, j=1,2, \ldots, n, \\
l_{0, n+j} y:=y^{(n+j-1)}(0)-(-1)^{j} y^{(n+j-1)}(1)=0, j=1,2, \ldots, n .
\end{gathered}
$$

Let $L_{0}$ be the operator of boundary-value problem (4)-(6),

$$
L_{0} y:=(-1)^{n} y^{(2 n)}(x), y \in D\left(L_{0}\right), D\left(L_{0}\right):=\left\{y \in W_{2}^{2 n}(0,1): l_{0, j} y=0, j=1,2, \ldots, 2 n\right\} \text {. }
$$

Assumption $B_{1}: n=2 \beta+1, \beta \in \mathbb{N}$.

Remark 1. The following relations are true

$$
l_{0, j} \in W_{0}^{*}(0,1), l_{0, n+j} \in W_{1}^{*}(0,1), j=1,2, \ldots, n \text {. }
$$

Let us consider the eigenvalue problem for an operator $L_{0}$

$$
(-1)^{n} y^{(2 n)}(x)-\lambda y=0, l_{0, j} y=0, \lambda \in \mathbb{C} j=1,2, \ldots, 2 n .
$$

Theorem 1. Assume that the condition $B_{1}: n=2 \beta+1$ is fulfilled. Then, all non-zero eigenvalues of the operator $L_{0}$ are of an even multiplicity. 
Proof of Theorem 1. The roots $\rho_{j}$ of the characteristic equation $(-1)^{n} \rho^{2 n}=\lambda,|\arg \rho| \leq$ $\frac{1}{2 n} \pi$, which corresponds to the differential equation (7), are defined by the relations

$$
\left(\omega_{j}\right)^{2 n}=(-1)^{n}=-1, \omega_{1}=i, \omega_{j}=\omega_{1} \exp i \frac{1}{n} \pi(j-1), j=2,3, \ldots, n .
$$

Consider the fundamental system of solutions of the differential equation (7)

$$
\begin{gathered}
y_{j}(x, \rho):=e^{w_{j} \rho x}+e^{w_{j} \rho(1-x)} \in H_{0}, j=1,2, \ldots, n, \\
y_{n+j}(x, \rho):=e^{w_{j} \rho x}-e^{w_{j} \rho(1-x)} \in H_{1}, j=1,2, \ldots, n .
\end{gathered}
$$

Substituting the general solution

$$
y(x, \rho):=\sum_{p=1}^{2 n} c_{p} y_{p}(x, \rho), c_{p} \in \mathbb{R}, p=1,2, \ldots, 2 n,
$$

of differential equation (7) into the boundary conditions (5), (6), we obtain the equation for determining the eigenvalues of the operator $L_{0}$

$$
\Delta(\rho):=\operatorname{det}\left(l_{0, m} y_{q}\right)_{m, q=1}^{2 n}=0 .
$$

From the assumption $B_{1}$ and the relations (8), (9), we have the equalities

$$
\begin{gathered}
l_{0, n+j} y_{m}(x, \rho)=0, l_{0, j} y_{n+m}(x, \rho)=0, j, m=1,2, \ldots, n, \\
\Delta(\rho)=\rho^{n^{2}} \prod_{j=1}^{n}\left(\omega_{j}\right)^{n} \Delta_{0}^{2}(\rho)=(-1)^{\beta} i \rho^{n^{2}} \Delta_{0}^{2}(\rho)=0, \\
\Delta_{0}(\rho)=\rho^{\alpha} \operatorname{det}\left(\left(\omega_{m}\right)^{j-1}\left(1+(-1)^{j-1} e^{\omega_{m} \rho}\right)\right)_{j, m=1}^{n}, \alpha=\frac{1}{2} n(n-1) .
\end{gathered}
$$

Lemma 1. Assume that the condition $B_{1}$ is fulfilled. Then, the operator $L_{0}$ is self-adjoint. Proof of Lemma 1. From the equalities (5) for $j=s$ and from the equalities (6) for $n+j=$ $2 n-s-1$, we obtain

$$
y^{(s-1)}(0)=(-1)^{s} y^{(s-1)}(1), y^{(2 n-s)}(0)=(-1)^{s} y^{(2 n-s)}(1), y \in D\left(L_{0}\right), s=1,2, \ldots, n .
$$

Thus,

$$
y^{(2 n-s)}(1) y^{(s-1)}(1)-y^{(2 n-s)}(0) y^{(s-1)}(0)=0 .
$$

We multiply the obtained equalities by the numbers $(-1)^{s-1}$, and sum them up for $s=$ $1,2, \ldots, 2 n$.

The obtained result is to be substituted into the relation

$$
\begin{aligned}
\left((-1)^{n} y^{(2 n)}, y ; L_{2}(0,1)\right)= & \sum_{s=1}^{2 n}(-1)^{s-1}\left(y^{(2 n-s)}(1) y^{(s-1)}(1)-y^{(2 n-s)}(0) y^{(s-1)}(0)\right)+ \\
& +\left(y,(-1)^{n} y^{(2 n)} ; L_{2}(0,1)\right) .
\end{aligned}
$$

Thus,

$$
\left((-1)^{n} y^{(2 n)}, y ; L_{2}(0,1)\right)=\left(y,(-1)^{n} y^{(2 n)} ; L_{2}(0,1)\right), y \in D\left(L_{0}\right)
$$


Therefore, the operator $L_{0}$ is self-adjoint and there exists a numerical sequence $\left\{\rho_{q}\right\}_{q=\overline{0, \infty}}$ of roots of the equation (11), which are numbered in ascending order and lie on the half-line $\operatorname{Im} \rho=0, \operatorname{Re} \rho \geq 0$.

Let $\lambda_{q}=\left(\rho_{q}\right)^{2 n}$ be corresponding eigenvalues of the operator $L_{0}, q=0,1, \ldots$.

We determine a system of eigenfunctions of $L_{0}$. Using the elements of systems (8), (9), we construct the functions

$$
\begin{aligned}
& v_{0, q}(x):=\theta_{0, q}\left\|\begin{array}{ccc}
y_{1}\left(x, \rho_{q}\right) & \ldots & y_{n}\left(x, \rho_{q}\right) \\
l_{0,2} y_{1} & \ldots & l_{0,2} y_{n} \\
\ldots & \ldots & \ldots \\
l_{0, n} y_{1} & \ldots & l_{0, n} y_{n}
\end{array}\right\|, q=1,2, \ldots \\
& v_{0, q}(x):=\theta_{1, q}\left\|\begin{array}{ccc}
y_{1}\left(x, \rho_{q}\right) & \ldots y_{m}\left(x, \rho_{q}\right) \ldots & y_{n}\left(x, \rho_{q}\right) \\
\omega_{1}\left(1-e^{\omega_{1} \rho_{q}}\right) & \ldots \omega_{m}\left(1-e^{\omega_{m} \rho_{q}}\right) \ldots & \omega_{n}\left(1-e^{\omega_{n} \rho_{q}}\right) \\
\ldots & \ldots & \ldots \\
\left(\omega_{1}\right)^{n-1}\left(1+e^{\omega_{1} \rho_{q}}\right) & \ldots\left(\omega_{m}\right)^{n-1}\left(1+e^{\omega_{m} \rho_{q}}\right) \ldots & \left(\omega_{n}\right)^{n-1}\left(1+e^{\omega_{n} \rho_{q}}\right)
\end{array}\right\|
\end{aligned}
$$

$q=1,2, \ldots$.

We choose the parameters $\theta_{1, q}$ so that $\left\|v_{0, q}(x) ; L_{2}(0,1)\right\|=1, q=1,2, \ldots$.

Analogically, we determine the eigenfunctions $v_{1, q}(x) \in L_{2}(0,1)$ of the operator

$$
\begin{aligned}
& v_{1, q}(x):=\theta_{2, q}\left\|\begin{array}{ccc}
y_{n+1}\left(x, \rho_{q}\right) & \ldots & y_{2 n}\left(x, \rho_{q}\right) \\
l_{0, n+2} y_{1} & \ldots & l_{0, n+2} y_{n} \\
\ldots & \ldots & \ldots \\
l_{0,2 n} y_{1} & \ldots & l_{0, n} y_{n}
\end{array}\right\|, q=0,1, \ldots \\
& v_{1, q}(x):=\theta_{3, q}\left\|\begin{array}{clc}
y_{n+1}\left(x, \rho_{q}\right) & \ldots & y_{2 n}\left(x, \rho_{q}\right) \\
\left(\omega_{1}\right)^{n+1}\left(1-e^{\omega_{1} \rho_{q}}\right) & \ldots & \left(\omega_{n}\right)^{n+1}\left(1-e^{\omega_{n} \rho_{q}}\right) \\
\ldots & \ldots & \ldots \\
\left(\omega_{1}\right)^{2 n-1}\left(1+e^{\omega_{1} \rho_{q}}\right) & \ldots & \left(\omega_{n}\right)^{2 n-1}\left(1+e^{\omega_{n} \rho_{q}}\right)
\end{array}\right\|, q=0,1, \ldots
\end{aligned}
$$

We choose the parameters $\theta_{3, q}$ so that $\left\|v_{1, q}\left(x, L_{0}\right) ; L_{2}(0,1)\right\|=1, q=0,1, \ldots$

The normed eigenfunctions of the operator $L_{0}$ which correspond to the eigenvalue $\lambda_{0}=0$ are determined by the formula

$$
v_{1,0}(x)=\sqrt{3}(2 x-1), v_{2,0}(x)=\sqrt{7}(2 x-1)^{3}, \ldots, v_{\beta, 0}(x):=\sqrt{4 \beta-1}(2 x-1)^{2 \beta-1} .
$$

It follows from the self-adjointness of the operator $L_{0}$ that the system of the functions

$$
V\left(L_{0}\right):=\left\{v_{j, q}, v_{s, 0}\left(x, L_{0}\right) \in L_{2}(0,1), s=1,2, \ldots, \beta, j=0,1, q=1,2, \ldots\right\}
$$

forms an orthonormal basis of the space $L_{2}(0,1)$.

Remark 2. The systems of functions $V_{1}\left(L_{0}\right):=\left\{v_{1, q}(x) \in L_{2}(0,1), q=1,2, \ldots\right\}, V_{0}\left(L_{0}\right):=$ $\left\{v_{m, 0}(x), v_{0, q}(x) \in L_{2}(0,1), m=1,2, \ldots, \beta, q=1,2, \ldots\right\}$ form orthonormal bases of the spaces $H_{0}, H_{1}$, respectively. 
Define the determinants (12), (13) using the relations

$$
\begin{aligned}
& v_{0, q}(x):=\theta_{1, q} \sum_{m=1}^{n} \Delta_{1, m}^{0}\left(\rho_{q}\right) y_{m}\left(x, \rho_{q}\right), \\
& v_{1, q}(x):=\theta_{3, q} \sum_{m=1}^{n} \Delta_{1, m}^{1}\left(\rho_{q}\right) y_{n+m}\left(x, \rho_{q}\right), \quad \Delta_{1, m}^{0}\left(\rho_{q}\right):= \\
& \equiv\left\|\begin{array}{cccc}
\omega_{1}\left(1-e^{\omega_{1} \rho_{q}}\right) & \ldots \omega_{m-1}\left(1-e^{\omega_{m-1} \rho_{q}}\right) & \omega_{m+1}\left(1-e^{\omega_{m+1} \rho_{q}}\right) \ldots & \omega_{n}\left(1-e^{\omega_{n} \rho_{q}}\right) \\
\omega_{1}^{2}\left(1+e^{\omega_{1} \rho_{q}}\right) & \ldots \omega_{m-1}^{2}\left(1+e^{\omega_{m-1} \rho_{q}}\right) & \omega_{m+1}^{2}\left(1+e^{\omega_{m+1} \rho_{q}}\right) \ldots & \omega_{n}^{2}\left(1+e^{\omega_{n} \rho_{q}}\right) \\
\ldots & \ldots & \ldots & \ldots \\
\omega_{1}^{n-1}\left(1+e^{\omega_{1} \rho_{q}}\right) & \ldots \omega_{m-1}^{n-1}\left(1+e^{\omega_{m-1} \rho_{q}}\right) & \omega_{m+1}^{n-1}\left(1+e^{\omega_{m+1} \rho_{q}}\right) \ldots & \omega_{n}^{n-1}\left(1+e^{\omega_{n} \rho_{q}}\right)
\end{array}\right\|, \\
& \Delta_{1, m}^{1}\left(\rho_{q}\right)=(-1)^{m-1} \Delta_{1, m}^{0}\left(\rho_{q}\right), m=1,2, \ldots, n, q=1,2, \ldots
\end{aligned}
$$

Let

$$
\begin{gathered}
y_{1, m}\left(x, \rho_{q}\right):=\omega_{m}(1-2 x) y_{n+m}\left(x, \rho_{q}\right) \in H_{0}, m=1,2, \ldots, n, \\
y_{1, n+m}\left(x, \rho_{q}\right):=\omega_{m}(1-2 x) y_{m}\left(x, \rho_{q}\right) \in H_{1}, m=1,2, \ldots, n, \\
y_{2,1}\left(x, \rho_{q}\right):=\sum_{j=1}^{n} \Delta_{1, j}^{0}\left(\rho_{q}\right) y_{1, j}\left(x, \rho_{q}\right), y_{2, m}\left(x, \rho_{q}\right):=y_{m}\left(x, \rho_{q}\right) m=2,3, \ldots, n,
\end{gathered}
$$

By means of direct calculation we make sure that the following relations are true

$$
L_{0} y_{2,1}\left(x, \rho_{q}\right)=\lambda_{q} y_{2,1}\left(x, \rho_{q}\right)+\xi_{0, q} v_{0, q}(x), \xi_{0, q}=-4 n\left(\rho_{q}\right)^{2 n-1}\left(\theta_{1, q}\right)^{-1}, \quad q=1,2, \ldots, .
$$

4. Nonlocal boundary-value problems. For arbitrary $p \in\{1,2, \ldots, n\}, k \in\{0,1, \ldots$, $2 n-1\}, b \in \mathbb{R}$, we consider the boundary-value problem

$$
\begin{gathered}
(-1)^{n} y^{(2 n)}(x)=f(x), x \in(0,1), \\
l_{1, j} y:=y^{(j-1)}(0)-(-1)^{j} y^{(j-1)}(1)=0, j \neq p, j=1,2, \ldots, n, \\
l_{1, p} y:=y^{(p)}(0)-(-1)^{p} y^{(p)}(1)+l_{p, k}^{1} y=0, \\
l_{1, n+j} y:=y^{(n+j-1)}(0)-(-1)^{j} y^{(n+j-1)}(1)=0, j=1,2, \ldots, n,
\end{gathered}
$$

where

$$
l_{p, k}^{1} y:=b\left(y^{(k-1)}(0)+(-1)^{p} y^{(k-1)}(1)\right) .
$$

Let $L_{1}:=L_{1, p, k, b}$ be the operator of the problem (17)-(21),

$L_{1} y:=(-1)^{n} y^{(2 n)}(x), y \in D\left(L_{1}\right), D\left(L_{1}\right):=\left\{y \in W_{2}^{2 n}(0,1): l_{1, j} y=0, j=1,2, \ldots, 2 n\right\}$, $V\left(L_{1}\right)$ be the system of root functions of the operator $L_{1}$.

For any eigenvalue $\lambda_{q}$ of the operator $L_{1}$ and the corresponding eigenfunction $v_{0, q}\left(x, L_{1}\right)$, the function $v_{1, q}\left(x, L_{1}\right) \in D\left(L_{1}\right)$, will be called the root function of the operator $L_{1}$; this function for some $c \in \mathbb{C}$ is a solution of the differential equation

$$
(-1)^{n} y^{(2 n)}(x)-\lambda_{q} y(x)=c v_{0, q}\left(x, L_{1}\right), q=0,1, \ldots .
$$

Similarly, the root functions of other orders are defined by

$$
(-1)^{n} v_{j+1, q}^{(2 n)}\left(x, L_{1}\right)-\lambda_{q} v_{j+1, q}\left(x, L_{1}\right)=c v_{j, q}\left(x, L_{1}\right), j>1, j \in^{N}, q=1,2, \ldots .
$$


Theorem 2. For any $p \in\{1,2, \ldots, n\}, k \in\{0,1, \ldots, 2 n-1\}, b \in \mathbb{R}$,

1) the eigenvalues of the operators $L_{0}$ and $L_{1}$ coincide,

2) the system of functions $V\left(L_{1}\right)$ is complete and minimal in the space $L_{2}(0,1)$.

Proof of Theorem 2. We show that the eigenvalues of the operators $L_{0}, L_{1}$ coincide.

Substituting the general solution of the differential equation (10) into boundary conditions (18)-(21), we obtain a system of linear equations of $2 n$ order whose matrix of coefficients has a minor of order $n$ all elements $l_{p, k}^{1} y_{n+j}(x, \rho)$ of which are equal to zero.

Thus,

$$
\operatorname{det}\left(l_{1, j} y_{m}\right)_{j, m=\overline{1,2 n}}=\operatorname{det}\left(l_{0, j} y_{m}\right)_{j, m=\overline{1, n}} \operatorname{det}\left(l_{0, n+j} y_{n+m}\right)_{j, m=\overline{1, n}}=\operatorname{det}\left(l_{0, j} y_{m}\right)_{j, m=\overline{1,2 n}} .
$$

We define the root functions of the operator $L_{1}$. By means of direct verification, one can make sure that $v_{m, 0}(x), v_{0, q}(x) \in D\left(L_{1}\right), m=1,2, \ldots, \beta, q=1,2, \ldots$.

Thus, the operator $L_{1}$ has the eigenfunctions

$$
v_{m, 0}\left(x, L_{1}\right):=v_{m, 0}(x), v_{0, q}\left(x, L_{1}\right):=v_{0, q}(x), m=1,2, \ldots, \beta, q=1,2, \ldots .
$$

We define the rows of the square matrix $\mathrm{B}_{p}\left(x, \rho_{q}\right):=\left(\beta_{m, s}^{p}\right)_{m, s=1}^{n}$ of the order $n$ by the following relations: the $p$-th row consists of the functions $\left\{y_{2, s}\left(x, \rho_{q}\right), s=1,2, \ldots, n\right\}$.

Elements of other rows are the following

$$
\beta_{m, s}^{p}\left(\rho_{q}\right):=\left(\rho_{q}\right)^{1-m} l_{0, m} y_{2, s}\left(x, \rho_{q}\right), m \neq p, m=1,2, \ldots, n .
$$

Taking into account the formulas (15)-(16), we obtain the relations

$$
\begin{gathered}
\beta_{p, s}^{p}\left(\rho_{q}\right)=2 \omega_{s}^{p-1}\left(1-(-1)^{p-1} e^{\omega_{s} \rho_{q}}\right)+2(p-1) \omega_{s}^{p-2}\left(\rho_{q}^{-1}\right)\left(1+(-1)^{p-1} e^{\omega_{s} \rho_{q}}\right), m=p, \\
\beta_{m, s}^{p}\left(\rho_{q}\right)=2 \omega_{s}^{m-1}\left(1+(-1)^{m-1} e^{\omega_{s} \rho_{q}}\right), m \neq p, m, s=1,2, \ldots n, q=1,2, \ldots,
\end{gathered}
$$

Let

$$
\begin{gathered}
y_{3, p}\left(x, \rho_{q}\right):=\operatorname{det} \mathrm{B}_{p}\left(x, \rho_{q}\right), \\
\Delta_{0, p}\left(\rho_{q}\right):=l_{1, p} y_{3, p}\left(x, \rho_{q}\right)=l_{0, p} y_{3, p}\left(x, \rho_{q}\right) .
\end{gathered}
$$

Substituting the function (23) into the boundary conditions (18)-(21), we obtain the relations

$$
l_{1, j} y_{3, p}\left(x, \rho_{q}\right)=l_{0, j} y_{3, p}\left(x, \rho_{q}\right)=0, j \neq p, j, p=1,2, \ldots, n, q=1,2, \ldots
$$

The root function of the operator $L_{1}$ is defined by the formula

$$
v_{1, q}\left(x, L_{1}\right):=v_{1, q}(x)+c_{p, k}\left(\rho_{q}\right) y_{3, p}\left(x, \rho_{q}\right), q=1,2, \ldots
$$

Substituting this expression into the boundary condition (19) we determine the unknown parameter

$$
c_{p, k}\left(\rho_{q}\right)=-l_{p, k}^{1} v_{1, q}(x) \Delta_{0, p}^{-1}\left(\rho_{q}\right), q=1,2, \ldots
$$


Remark 3. If $q \rightarrow \infty$, then for the sequence $\Delta_{0, p}\left(\rho_{q}\right)$ we have the relation

$$
\left|\Delta_{0, p}\left(\rho_{q}\right)\right|=\left(W\left(1, \omega_{2}, \ldots, \omega_{n}\right)+e^{i \rho_{q}} W\left(-1, \omega_{2}, \ldots, \omega_{n}\right)\right)\left(1+O\left(q^{-1}\right)\right),
$$

here $W\left(\omega_{1}, \omega_{2}, \ldots, \omega_{n}\right)$ is the Vandermonde determinant of the order $0,1, \ldots, n-1$ constructed on the basis of the numbers $\omega_{1}, \omega_{2}, \ldots, \omega_{n}$.

Consequently, the inequality holds

$$
0<C_{1} \leq\left|\Delta_{0, p}\left(\rho_{q}\right) \rho_{q}^{1-p}\right| \leq C_{2}<\infty, q=1,2, \ldots
$$

Taking into account the formula (13), we obtain the inequality

$$
\left|c_{p, k}\left(\rho_{q}\right)\right| \leq C_{3}|b| \rho_{q}^{k-p}, q=1,2, \ldots,
$$

These root functions of the operator $L_{1}$ are determined by the formulas (22)-(25). Thus, the operator $L_{1}$ has functions which are root functions in the sense of the following equalities

$$
L_{1} v_{0, q}\left(x, L_{1}\right)(x)=\lambda_{q} v_{0, q}\left(x, L_{1}\right) L_{1} v_{1, q}\left(x, L_{1}\right)(x)-\lambda_{q} v_{1, q}\left(x, L_{1}\right)=\xi_{p, k, q} v_{0, q}\left(x, L_{1}\right),
$$

$\left|\xi_{p, k, q}\right| \leq C_{4} \rho_{q}^{2 n-1+k-p}, q=1,2, \ldots$.

There is an adjoint problem for the problem (17)-(21).

From the root functions of the operator of the adjoint problem, one can construct a system $W\left(L_{1}\right)$ which is biorthogonal to the systems $V\left(L_{1}\right)$.

Therefore, the system $V\left(L_{1}\right)$ is complete and minimal in the space $L_{2}(0,1)$.

5. Transformation operators. Let $G:=\left\{g_{q}(x)\right\}_{q=1}^{\infty}$ be a sequence of functions from the set $C^{\infty}[0,1]$ with the property

$$
g_{q}(x) \equiv g_{q}(1-x), g_{q}^{(m)}(x) \equiv(-1)^{m} g_{q}^{(m)}(1-x), x \in[0,1], q, m=1,2, \ldots
$$

We consider the system $Z(G)$ of the functions

$$
\begin{gathered}
z_{m, 0}(x):=v_{m, 0}(x), m=1,2, \ldots, \beta, z_{0, q}(x):=v_{0, q}(x), z_{1, q}(x):=v_{1, q}(x)+g_{q}(x), \\
q=1,2, \ldots
\end{gathered}
$$

For each system $Z(G)$ in the space $L_{2}(0,1)$, we determine an operator $R(G):=E+S(G)$ that maps the elements of the system $V\left(L_{0}\right)$ into the corresponding elements of the system $Z(G)$

$z_{m, 0}(x):=R(G) v_{m, 0}(x), m=1,2, \ldots, \beta, z_{m, q}(x):=R(G) v_{m, q}(x), m=0,1, q=1,2, \ldots$

Let $\Gamma\left(L_{0}\right)$ be the set of all the possible systems $Z(G)$ whose elements are determined by the formula (29), $Q\left(L_{0}\right)$ be the set of the operators $R(G), Q_{c}\left(L_{0}\right):=Q\left(L_{0}\right) \cap\left[L_{2}(0,1)\right]$.

Lemma 2. Any system of the functions $Z(G)$ whose elements are determined by formula (28), (29) is complete and minimal in the space $L_{2}(0,1)$. 
Proof of Lemma 2. We shall prove by contradiction that $Z(G)$ is complete in the space $L_{2}(0,1)$.

Let there exist a function $h \in L_{2}(0,1), h=h_{0}+h_{1}, h_{j} \in H_{j}, j=0,1$ with the property

$$
\left(h, z_{k, q}(x) ; L_{2}(0,1)\right)=0, k=1,2, \ldots, \beta, q=0, k=0,1, q=1,2, \ldots .
$$

Taking into account the formula (29) and Remark 2, we obtain $h_{0}=0, h=h_{1}$. Then, from the definition of functions (29) and assumption (30), we deduce the equality

$$
\left(h, z_{1, q}(x) ; L_{2}(0,1)\right)=\left(h, v_{1, q}(x) ; L_{2}(0,1)\right)=0, q=1,2, \ldots .
$$

Applying Remark 2, we obtain $h \equiv 0$.

Thus, the system $Z(G)$ is complete in $L_{2}(0,1)$.

For any operators $R\left(G_{j}\right)=E+S\left(G_{j}\right) \in Q\left(L_{0}\right)$ we get $S\left(G_{j}\right): H_{1} \rightarrow L_{2}(0,1)$, $S\left(G_{j}\right): L_{2}(0,1) \rightarrow 0, S\left(G_{1}\right) S\left(G_{2}\right)=S\left(G_{2}\right) S\left(G_{1}\right)=0$.

On the set $Q\left(L_{0}\right)$ we define the operation of multiplication

$$
\left.R G_{2}\right) R\left(G_{1}\right)=\left(E+S\left(G_{1}\right)\right)\left(E+S\left(G_{2}\right)\right)=E+S\left(G_{1}\right)+S\left(G_{2}\right) .
$$

From the definition for the operator $S(G): H_{1} \rightarrow L_{2}(0,1), S(G): L_{2}(0,1) \rightarrow 0$, we deduce $S^{2}(G)=0$.

Therefore, $(E+S(G))(E-S(G))=E$, and there exists an operator $R(G)^{-1}=E-S(G)$.

Consequently, the system $Z(G)$ of functions is minimal in the space $L_{2}(0,1)$.

Multiplication of operators is commutative. Therefore, the following Lemma is true.

Lemma 3. A set $Q\left(L_{0}\right)$ is the Abelian group of operators with respect to multiplication, which contains an Abelian group $Q_{c}\left(L_{0}\right)$.

Let $\left\{\phi_{q}\right\}_{q=1}^{\infty} \subset \mathbb{C}$ be the bounded sequence, $\left|\phi_{q}\right| \leq C_{5}<\infty, q=1,2, \ldots$.

We consider the particular case of a system $G: G_{\phi}:=\left\{g_{q}(x):=\phi_{q}(1-2 x) v_{1, q}(x), q=\right.$ $1,2, \ldots\}$.

Lemma 4. For any bounded sequence $\left\{\phi_{q}\right\} \subset \mathbb{C}$ the system $Z\left(G_{\phi}\right)$ is the Riesz basis in the space $L_{2}(0,1)$.

Proof of Lemma 4. We show that the operator $R\left(G_{\phi}\right)$ is bounded.

For arbitrary

$$
h=\sum_{j, q} h_{j, q} v_{j, q}(x) \in L_{2}(0,1), h_{j, q}=\left(h, v_{j, q}(x) ; L_{2}(0,1)\right), j=0,1, q=1,2, \ldots,
$$

we estimate the norm of the function $f=R\left(G_{\phi}\right) h$ in the space $L_{2}(0,1)$.

Thus,

$$
\begin{gathered}
f=\sum_{q}\left(h_{0, q} v_{j, q}(x)+h_{1, q}\left(1+\phi_{q}(1-2 x)\right) v_{1, q}(x)\right), \\
\left\|f ; L_{2}(0,1)\right\|^{2} \leq 6 \sum_{j, q} h_{j, q}{ }^{2}+4 C_{5}^{2}\left\|h ; L_{2}(0,1)\right\|^{2} \leq C_{6}^{2}\left\|h ; L_{2}(0,1)\right\|^{2}, C_{6}^{2}=6+4 C_{5}^{2}, \\
\left\|R\left(G_{\phi}\right) ;\left[L_{2}(0,1)\right]\right\| \leq C_{6} .
\end{gathered}
$$

Taking into account the equality $R^{-1}\left(G_{\phi}\right)=2 E-R\left(G_{\phi}\right) \in\left[L_{2}(0,1)\right]$ and N. K. Bari theorem $([9])$ we obtain statement of the lemma. 
6. Boundary value problem with regular according to Birkhoff conditions. Let $k=p$,

$$
l_{p, p}^{1} y:=b\left(y^{(p-1)}(0)+(-1)^{p} y^{(p-1)}(1)\right),
$$

$L_{2}:=L_{2, p, b}$ be the operator of the problem (17)-(20), (31); $V\left(L_{2}\right)$ be the system of root functions of the operator $L_{2}$, which are determined by the relations

$$
\begin{gathered}
v_{m, 0}\left(x, L_{2}\right):=v_{m, 0}(x), v_{0, q}\left(x, L_{2}\right):=v_{0, q}(x), m=1,2, \ldots, \beta, q=1,2, \ldots, \\
v_{1, q}\left(x, L_{2}\right):=v_{1, q}(x)+c_{p, p}\left(\rho_{q}\right) y_{3, p}\left(x, \rho_{q}\right), q=1,2, \ldots
\end{gathered}
$$

Substituting this expression into the boundary condition (19), we determine the unknown value

$$
c_{p, p}\left(\rho_{q}\right)=-l_{p}^{1} v_{1, q}(x) \Delta_{0, p}^{-1}\left(\rho_{q}\right), q=1,2, \ldots
$$

Theorem 3. For arbitrary $p \in\{1,2, \ldots, n\}, b \in \mathbb{R}$, the system $V\left(L_{2}\right)$ is a Riesz basis in the space $L_{2}(0,1)$.

Proof of Theorem 3. Taking into account the inequality (27), we obtain the relationship $\left|c_{p, p}\left(\rho_{q}\right)\right| \leq C_{3}|b|$.

Let $\beta^{1}\left(\rho_{q}\right):=\operatorname{det}\left(\beta_{m, s}\left(\rho_{q}\right)\right)_{m, s=2}^{n}, \beta_{m, s}:=2 \omega_{s}^{m-1}\left(1+(-1)^{m-1} \exp \omega_{s} \rho_{q}\right)$. We determine the numbers $c_{p}^{1}\left(\rho_{q}\right)$ by means of the equality

$$
c_{p}^{1}\left(\rho_{q}\right)=c_{p, p}\left(\rho_{q}\right) \beta^{1}\left(\rho_{q}\right) .
$$

Let $\phi_{q}=c_{1, p}\left(\rho_{q}\right), g_{q}(x):=c_{1, p}\left(\rho_{q}\right) v_{1, q}(x), q=1,2, \ldots$ Taking into account the inequality (27), we obtain the inequality $\left|c_{p}^{1}\left(\rho_{q}\right)\right| \leq C_{3}|b|$.

Taking into account Lemma 4 , we obtain the assertion: $Z\left(G_{\phi}\right)$ is the Riesz basis in this space $L_{2}(0,1)$.

Consider the sequence of functions

$$
\psi_{p}\left(x, \rho_{q}\right)=c_{p} y_{3, p}\left(x, \rho_{q}\right)-c_{p}^{1} v_{1, q}(x) .
$$

Taking into account the expansions of the functions $y_{3, p}\left(x, \rho_{q}\right), y_{1, p}\left(x, \rho_{q}\right), v_{1, q}(x)$ and the equality (35) for the functions $\psi_{p}\left(x, \rho_{q}\right)$, we obtain the formulas

$$
\begin{gathered}
v_{1, q}\left(x, L_{2}\right)=v_{1, q}(x)+\psi_{p}\left(x, \rho_{q}\right)+c_{p}^{1}\left(\rho_{q}\right) v_{1, q}(x), \\
\psi_{p}\left(x, \rho_{q}\right)=\sum_{m=2}^{n} \alpha_{0, m, p}\left(\rho_{q}\right) y_{m}\left(x, \rho_{q}\right)+\alpha_{1, m, p}\left(\rho_{q}\right)(1-2 x) y_{n+m}\left(x, \rho_{q}\right),
\end{gathered}
$$

where $\alpha_{0, m, p}\left(\rho_{q}\right), \alpha_{1, m, p}\left(\rho_{q}\right)$ are bounded sequences.

Taking into account the definition of the numbers $\omega_{1}, \ldots, \omega_{n}$, we obtain the inequality

$$
\sum_{q=1}^{\infty}\left\|\psi_{p}\left(x, \rho_{q}\right) ; L_{2}(0,1)\right\|^{2}<\infty .
$$

Thus, the complete system of functions $V\left(L_{2}\right)$ in the space $L_{2}(0,1)$ is quadratically close to the Riesz basis $Z\left(G_{\phi}\right)$ in this space. Taking into account N. K. Bary's theorem (see [9]), we obtain the assertion of the theorem. 
Let

$$
g_{3, q}(x):=v_{1, q}\left(x, L_{2}\right)-v_{1, q}(x), q=1,2, \ldots .
$$

For any sequence of the numbers $\left\{\varphi_{q}\right\}_{q=1}^{\infty}$, consider the system $G_{\varphi}:=\left\{g_{3, q}(x), q=1,2, \ldots\right\}$ of functions, the corresponding transformation operator $R\left(G_{\varphi}\right)=E+S\left(G_{\varphi}\right) \in Q\left(L_{0}\right)$ and the system

$$
Z(\varphi):=\left\{z_{m, q}(x) \in L_{2}(0,1): z_{m, 0}:=v_{m, 0}, z_{0, q}:=v_{0, q}, z_{1, q}:=v_{1, q}+\varphi_{q} g_{3, q}, q=1,2, \ldots\right\} .
$$

Lemma 5. For any bounded sequence $\left\{\varphi_{q}\right\} \subset \mathbb{C}$ the system $Z(\varphi)$ is the Riesz basis in the space $L_{2}(0,1)$.

Proof of Lemma 5. Let $\left|\varphi_{q}\right| \leq C_{7}<\infty, q=1,2, \ldots$. Then for any $h \in L_{2}(0,1), f:=$ $R\left(Z_{\varphi}\right) h$ the inequality is true

$$
C_{8}\left\|h ; L_{2}(0,1)\right\|^{2} \leq \sum_{m, k}\left|\left(h, v_{m, k}\left(x, L_{2}\right) ; L_{2}(0,1)\right)\right|^{2} \leq C_{9}\left\|h ; L_{2}(0,1)\right\|^{2} .
$$

Thus,

$$
\sum_{m, k}\left|\left(h, z_{m, k}(x) ; L_{2}(0,1)\right)\right|^{2} \leq \mathrm{C}_{10}\left\|h ; L_{2}(0,1)\right\|^{2}, \quad \mathrm{C}_{10}=2\left(C_{9}+1\right)^{2}\left\|R\left(L_{2}\right) ;\left[L_{2}(0,1)\right]\right\|^{2} .
$$

So, the operators $R^{*}\left(Z_{\varphi}\right), R^{*}\left(Z_{\varphi}\right)^{-1}: L_{2}(0,1) \rightarrow L_{2}(0,1)$ are bounded.

Taking into account the theorem of N. K. Bary (see [9]), we obtain the assertion: biorthogonal system of functions $W\left(L_{2}\right)$ is the basis of the space $L_{2}(0,1)$.

Therefore, the system of functions $V\left(L_{2}\right)$ is a basis of the space $L_{2}(0,1)$.

\section{Multipoint problems.}

7.1. Multipoint problem for a differential equation. Consider the multipoint problem

$$
\begin{gathered}
(-1)^{n} y^{(2 n)}(x)=f(x), x \in(0,1), \\
l_{3, j} y:=y^{(j-1)}(0)-(-1)^{j} y^{(j-1)}(1)=0, j \neq p, j=1,2, \ldots, n, \\
l_{3, p} y:=y^{(p-1)}(0)-(-1)^{p} y^{(p-1)}(1)+l_{p}^{2} y=0, \\
l_{3, n+j} y:=y^{(n+j-1)}(0)-(-1)^{j} y^{(n+j-1)}(1)=0, j=1,2, \ldots, n,
\end{gathered}
$$

where

$$
l_{p}^{2} y:=\sum_{j=0}^{r} \sum_{m=0}^{k_{p}} b_{p, m, j} y^{(m)}\left(\left(x_{j}\right)\right) .
$$

Consider the following assumptions.

Assumption $B_{2}: x_{j}=1-x_{r-j}, b_{p, m, j}=(-1)^{m} b_{p, m, r-j}, j=0,1, \ldots, r, m=0,1, \ldots, k_{p}$, $p=1,2, \ldots, n$.

Assumption $B_{3}: k_{p} \leq p-1, p=1,2, \ldots, n$.

Let $L_{3}:=L_{3, p}$ be the operator of the problem (36)-(40),

$$
L_{3} y:=(-1)^{n} y^{(2 n)}(x), y \in D\left(L_{3}\right), D\left(L_{3}\right):=\left\{y \in W_{2}^{2 n}(0,1): l_{3, j} y=0, j=1,2, \ldots, 2 n\right\},
$$

$V\left(L_{3}\right)$ be the system of root functions of the operator $L_{3}$. 
Theorem 4. Suppose that the assumption $B_{1}, B_{2}$ holds. Then for arbitrary $p \in\{1,2, \ldots$, $n\}, x_{j} \in[0,1), b_{p, m, j} \in \mathbb{R}:$

1) the eigenvalues of the operators $L_{0}$ and $L_{3}$ coincide;

2) the system of functions $V\left(L_{3}\right)$ is complete and minimal in the space $L_{2}(0,1)$;

3) if an addition the assumption $B_{3}$ holds, then the system $V\left(L_{3}\right)$ is a Riesz basis in the space $L_{2}(0,1)$.

Proof of Theorem 4. The first assertion of the theorem is established by the arguments of Theorem 2. By direct verification we see that

$$
v_{m, 0}(x), v_{0, q}(x) \in D\left(L_{3}\right), m=1,2, \ldots, \beta, q=1,2, \ldots .
$$

Therefore, the operator $L_{3}$ has its eigenfunctions

$$
v_{m, 0}\left(x, L_{3}\right):=v_{m, 0}(x), v_{0, q}\left(x, L_{3}\right):=v_{0, q}(x), \quad m=1,2, \ldots, \beta, q=1,2, \ldots .
$$

The root functions are defined as the sum

$$
v_{1, q}\left(x, L_{3}\right):=v_{1, q}(x)+c_{p}^{2}\left(\rho_{q}\right) y_{3, p}\left(x, \rho_{q}\right)
$$

Substituting this expression into conditions (39), we determine the unknown parameter $c_{p}^{2}\left(\rho_{q}\right)$

$$
c_{p}^{2}\left(\rho_{q}\right)=-l_{p}^{2} v_{1, q}\left(x, \rho_{q}\right) \Delta_{0, p}^{-1}\left(\rho_{q}\right),=1,2, \ldots,
$$

Thus, the operator $L_{3}$ it has the root functions (41) - (43), and the system of functions $V\left(L_{3}\right)$ is an element of the set $\Gamma\left(L_{0}\right)$.

Therefore, from Lemma 2, we obtain the second assertion of the theorem.

From the assumption $B_{3}$ we have the estimate $\left|l_{p}^{2} v_{1, q}\right| \leq \mathrm{C}_{11}\left(\rho_{\mathrm{q}}\right)^{\mathrm{k}_{\mathrm{p}}}, \quad 0<C_{11}<\infty$.

Taking into account the relation (26), we obtain the boundedness of the sequence $c_{2, p}\left(\rho_{q}\right)$.

Taking into account Lemma 5, we obtain the third statements of the theorem.

7.2. Multipoint problem for a differential equation with an involution. Consider the following multipoint problem for arbitrary fixed $s, p \in\{1,2, \ldots, n\}$

$$
\begin{gathered}
(-1)^{n} y^{(2 n)}(x)+a_{s}\left(y^{(2 s-1)}(x)+y^{(2 s-1)}(1-x)\right)=f(x), x \in(0,1), \\
l_{3, j} y:=y^{(j-1)}(0)-(-1)^{j} y^{(j-1)}(1)=0, j \neq p, j=1,2, \ldots, n, \\
l_{3, p} y:=y^{(p-1)}(0)-(-1)^{p} y^{(p-1)}(1)+l_{p}^{2} y=0, \\
l_{3, n+j} y:=y^{(n+j-1)}(0)+(-1)^{n+j} y^{(n+j-1)}(1)=0, j=1,2, \ldots, n,
\end{gathered}
$$

where

$$
l_{p}^{2} y=\sum_{j=0}^{r} \sum_{m=0}^{k_{p}} b_{p, m, j} y^{(m)}\left(x_{j}\right) .
$$

Let $L_{4}:=L_{4, p, s}$ be the operator of the problem (44)-(48),

$$
\begin{gathered}
L_{4} y:=(-1)^{n} y^{(2 n)}(x)+a_{s}\left(y^{(2 s-1)}(x)+y^{(2 s-1)}(1-x)\right), \\
y \in D\left(L_{4}\right), D\left(L_{4}\right):=\left\{y \in W_{2}^{2 n}(0,1): l_{3, j} y=0, j=1,2, \ldots, 2 n\right\},
\end{gathered}
$$

$V\left(L_{4}\right)$ be the system of root functions of the operator $L_{4}$.

Let $R\left(L_{4}\right)$ be the transformation operator mapping the system $V\left(L_{0}\right)$ into the system $V\left(L_{4}\right)$. 
Theorem 5. Suppose that the assumptions $B_{1}, B_{2}$ hold. Then for arbitrary $s, p \in\{1,2, \ldots$, $n\}, x_{j} \in[0,1), b_{p, m, j} \in \mathbb{R}, m=0,1, \ldots, k_{p}, m=0,1, \ldots, r$

1) the eigenvalues of the operators $L_{0}$ and $L_{4}$ coincide;

2) the system $V\left(L_{4}\right)$ is complete and minimal in space $L_{2}(0,1)$;

3) the system $V\left(L_{4}\right)$ is a Riesz basis in the space $L_{2}(0,1)$ iprovided that the assumption $B_{3}$ also holds.

Proof of Theorem 5. The first assertion of the theorem is established by considerations of Theorem 2. By direct verification we see that

$$
v_{m, 0}(x), v_{0, q}(x) \in D\left(L_{4}\right), m=1,2, \ldots, \beta, q=1,2, \ldots
$$

Thus, the operator $L_{4}$ has its eigenfunctions

$$
v_{m, 0}\left(x, L_{4}\right):=v_{m, 0}(x), v_{0, q}\left(x, L_{4}\right):=v_{0, q}(x), m=1,2, \ldots, \beta, q=1,2, \ldots
$$

Consider the functions

$$
\begin{gathered}
y_{4,1, s}\left(x, \rho_{q}\right):=\sum_{j=1}^{n} \alpha_{j, s}\left(\rho_{q}\right) \Delta_{1, j}^{0}\left(\rho_{q}\right) y_{1, j}\left(x, \rho_{q}\right), \\
y_{4, m, s}\left(x, \rho_{q}\right):=y_{m}\left(x, \rho_{q}\right) \in L_{2}(0,1), m=2,3, \ldots, n .
\end{gathered}
$$

We substitute the sum

$$
v_{1, q}(x)+y_{4,1, s}\left(x, \rho_{q}\right) .
$$

with indefinite coefficients $\alpha_{j, s}\left(\rho_{q}\right)$ into the equation

$$
(-1)^{n} y^{(2 n)}(x)+a_{s}\left(y^{(2 s-1)}(x)+y^{(2 s-1)}(1-x)\right)=\lambda_{q} y(x), x \in(0,1)
$$

To determine the parameters $\alpha_{j, s}\left(\rho_{q}\right)$, we obtain the equalities

$$
4 n\left(\rho_{q}\right)^{2 n-1} \alpha_{j, s}\left(\rho_{q}\right)+2 a_{s}\left(\rho_{q} \omega_{j}\right)^{2 s-1}=0 .
$$

Thus,

$$
\alpha_{j, s}\left(\rho_{q}\right)=-(2 n)^{-1} a_{s}\left(\rho_{q}\right)^{2 s-2 n} \omega_{j}^{2 s},
$$

In particular, for $s=n$ we have $\alpha_{j, n}\left(\rho_{q}\right)=(2 n)^{-1} a_{n}$,

$$
y_{4,1, n}\left(x, \rho_{q}\right):=(2 n)^{-1} a_{n} y_{2,1}\left(x, \rho_{q}\right)
$$

We define the rows of the square matrix $B_{1, p, s}\left(x, \rho_{q}\right):=\left(\beta_{j, m}^{p, s}\right)_{j, m=1}^{n}$ of the order $n$ by the formulas: the $p$-th row consists of the functions (50), (51), the elements of the other rows are the numbers $\beta_{j, m}\left(\rho_{q}\right):=\rho_{q}^{1-j} l_{0, j} y_{4, m}\left(x, \rho_{q}\right), j \neq p, j, m=1,2, \ldots, n$.

Remark 4. Taking into account the formulas (14), (16), we obtain the equalities

$$
\beta_{j, m}\left(\rho_{q}\right)=2 \omega_{m}^{j-1}\left(1+(-1)^{j-1} e^{\omega_{m} \rho_{q}}\right), j \neq p, j=1,2, \ldots, n, m=2,3, \ldots, n .
$$


Let

$$
y_{5, p, s}\left(x, \rho_{q}\right):=\left(\Delta_{1,1}^{0}\left(\rho_{q}\right)\right)^{-1} \operatorname{det} \mathrm{B}_{1, p, s}\left(x, \rho_{q}\right), \quad \Delta_{0, p, s}\left(\rho_{q}\right):=l_{0, p} y_{5, p, s}\left(x, \rho_{q}\right)
$$

By the substitution of expression (55) into the conditions (37)-(48), we obtain the relation

$$
l_{3, j} y_{5, p, s}\left(x, \rho_{q}\right)=l_{0, j} y_{5, p, s}\left(x, \rho_{q}\right) \delta_{j, p}=\Delta_{0, p, s}\left(\rho_{q}\right) \delta_{j, p}, j, p=1,2, \ldots, n,
$$

$\delta_{j, p}=0, j \neq p, \delta_{j, p}=1, j=p, j, p=1,2, \ldots, n$.

The root function of the operator $L_{4}$ is defined by the formula

$$
v_{1, q}\left(x, L_{4}\right):=v_{1, q}(x)+c_{0, p, s}\left(\rho_{q}\right) y_{3, p}\left(x, \rho_{q}\right)+y_{5, p, s}\left(x, \rho_{q}\right) .
$$

Substituting the expression (57) into the boundary condition (48), we determine the unknown value

$$
\begin{gathered}
c_{0, p, s}\left(\rho_{q}\right)=\Delta_{0, p}^{-1}\left(\rho_{q}\right)\left(-l_{p}^{2} v_{1, q}(x)+l_{0, p} y_{5, p, s}\left(x, \rho_{q}\right)\right), \\
q=1,2, \ldots .
\end{gathered}
$$

It follows from (49), (56) that the system of root functions $V\left(L_{4}\right)$ is an element of the set $\Gamma_{1}\left(L_{0}\right)$.

Therefore, from Lemma 1 we obtain the assertion of the theorem.

Suppose that the condition $s<n$ is satisfied. Then it follows from (53) that the systems of the functions $V\left(L_{4}\right)$ and $V\left(L_{3}\right)$ are quadratically close.

Therefore, taking into account Theorem 4, N. K. Bari's theorem (see [9]) and the completeness of the system, we obtain the assertion of the theorem.

In the case of $s=n$, the formula (54) yields the proportionality of the functions $y_{4,1, n}\left(x, \rho_{q}\right)$ and $y_{2,1}\left(x, \rho_{q}\right)$, therefore the further proof is carried out according to the scheme of Theorem 4 .

7.3. Multipoint problem for a differential equation of high order with an involution. Consider the multipoint problem (1)-(4). Let $L$ be the operator of the problem (1)-(4), $V(L)$ be the system of root functions of the operator $L$.

Theorem 6. Suppose that the assumptions $B_{1}, B_{2}$ hold. Then for arbitrary $x_{j} \in[0,1)$, $b_{j, m, s}, a_{j} \in \mathbb{R}, s=0,1, \ldots, r, m=0,1, \ldots, k_{j}, j=1,2, \ldots, n$

1) the eigenvalues of the operators $L_{0}$ and $L$ coincide;

2) the system $V(L)$ is complete and minimal in the space $L_{2}(0,1)$;

$3)$ the system $V(L)$ is a Riesz basis in the space $L_{2}(0,1)$ provided that the assumption $B_{3}$ also holds.

Proof of Theorem 6. The first assertion of the theorem is established by the arguments of Theorem 2.

Let $R(L)=E+S(L)$ be the operator the mapping $V\left(L_{0}\right) \rightarrow V(L)$,

$$
V(L) v_{j, q}(x, L):=R(L) v_{j, q}(x), j=0,1, . ., \beta, q=0, j=0,1 q=1,2, \ldots .
$$

We define the operator $R(L)=E+S(L)$ by the relations

$$
R(L)=\prod_{s=1}^{n} \prod_{p=1}^{n} R\left(L_{4, p, s}\right)=E+\sum_{s, p=1}^{n} S\left(L_{4, p, s}\right) .
$$


Taking into account the fact that the set $Q\left(L_{0}\right)$ is a group with respect to multiplication, we obtain the inclusions $R(L) \in Q\left(L_{0}\right)$.

Thus, according to Lemma 1 , the system $V(L)$ is complete and minimal in the space $L_{2}(0,1)$.

If the assumption $B_{3}$ is true, then, according to Theorem 5, each of the operators is bounded.

Therefore, $R(L), R^{-1}(L) \in\left[L_{2}(0,1)\right]$, i.e. according to N. K. Bari's theorem (see [9]), we obtain the assertion of Theorem 6 .

\section{REFERENCES}

1. Ya.O. Baranetskij, Boundary value problems with irregular conditions for differential-operator equations, Bukov. Mathemat. Journ., 3 (2015), №3-4, 33-40. (in Ukrainian)

2. Ya O. Baranetskij, A.A. Basha, Nonlocal multipoint problem for differential-operator equations of order 2n, J. Math. Sci., 217 (2016), №2, 176-186.

3. Ya.O. Baranetskij, P.I. Kalenyuk, L.I. Kolyasa, Boundary-value for abstract second-order differential equations with operators involution, Bukov. Mathemat. Journ., 4 (2016), №3-4, 22-30.

4. Ya.O. Baranetskij, P.I. Kalenyuk, L.I. Kolyasa, M.I. Kopach, The nonlocal problem for the differentialoperator equation of the even order with the involution, Carpathian Math. Publ., 9 (2017), №2, $109-119$.

5. Ya. Baranetskij, L. Kolyasa, Boundary-value problem for abstract second-order differential equation with involution, Journal Visnyk of Lviv Polytechnic National University. Physical and mathematical sciences, 871 (2017), 20-27. (in Ukrainian)

6. G.D. Birkhoff, On the asymptotic character of the solutions of certain linear differential equations containing a parameter, Trans. Amer. Math. Soc., 9 (1908), 219-231.

7. G.D. Birkhoff, Boundary value and expansion problems of ordinary linear differential equations, Trans. Amer. Math. Soc., 91 (1908), №4, 373-395.

8. A. Cabada, F.A.F. Tojo, Solutions and Green's function of the first order linear equation with reflection and initial conditions, Boundary Value Problems, 99 (2014), 1-16.

9. I.Ts. Gokhberg, M.G. Krein, Introduction to the Theory of Linear Not Self-Adjoint Operators, Nauka, Moscow, 1965, 448 p. (in Russian)

10. N.I. Ionkin, Solution of a boundary-value problem in heat conduction with a nonclassical boundary condition, Differ. Equat., 13 (1977), №2, 204-211.

11. P.I. Kalenyuk, Ya.E. Baranetskii, Z.N. Nitrebich, Generalized Method of the Separation of Variables, Naukova Dumka, Kiev, 1993, 231 p. (in Russian)

12. P.I. Kalenyuk, Ya.O. Baranetskij, L.I. Kolyasa, Nonlocal boundary value problems for the operator of differentiation of even order, Nonclassical problems of the theory of differential equations: A collection of scientific papers is dedicated to the 80th birthday of Bogdan Iosifovich Ptashnik, 2017, 91-110. (in Ukrainian)

13. G.M. Kesel'man, On the unconditional convergence of eigenfunction expansions of certain differential operators, Izv. Vyssh. Uchebn. Zaved. Mat., 39 (1964), 82-93. (in Russian)

14. V.P. Mikhailov, On Riesz bases in L2(0;1), Doklady Akad. Nauk SSSR, 144 (1962), 981-984. (in Russian)

15. A.Yu. Mokin, On the of initial boundary value problems for the heat equation, Differ. Equat., 45 (2009), №1, 126-141.

16. M.A. Naimark, Linear differential operators, Nauka, Moscow, 1969, 528 p. (in Russian)

17. D.O'Regan, Existence results for differential equations with reflection of the argument, J. Aust-tral. Math. Soc., 57 (1994), №2, 237-260.

18. B.Yo. Ptashnyk, V.S. Il'kiv, I.Ya. Kmit', V.M. Polishchuk, Nonlocal boundary problems for partial differential equations, Naukova Dumka, Kyiv, 2002, 416 p. (in Ukrainian)

19. A.A. Shkalikov, On the basis problem of the eigenfunctions of an ordinary differential operator, Uspekhi Mat. Nauk, 34 (1979), №5, 235-236. 
20. M.H. Stone, A comparison of the series of Fourier and Birkhoff, Trans. Amer. Math. Soc. 28 (1926), №4, 695-761.

21. M.H. Stone, Irregular differential systems of ordinary two and the related expansion problems, Trans. Amer. Soc., 29 (1927), №1, 23-53.

22. J. Tamarkin, Sur quelques points de la théorie des equations différentielles linéaires ordinaires et sur la généralisation de la serie de Fourier, Rend. Circ. Matem. Palerm., (1912), №3, 345-382.

23. J. Tamarkin, Some general problems of the theory of ordinary linear differential equations and expansions of an arbitrary function in series of fundamental functions, Math. Z., (1927), 1-54.

Lviv Polytechnic National University

Lviv, Ukraine

kolyasa.lubov@gmail.com 